\title{
Fetus In Fetu: caso clínico estudiado con tomografía tridimensional
}

\author{
Fetus in Fetu: clinical case studied with three-dimensional tomography \\ Gladys Hilda Virginia Ordóñez,, Carlos Fernando Rosales Ordoñez, ${ }^{2}$ Enrique Antonio Tomé, ${ }^{3}$ Carlos Almílcar Godoy Mejía, ${ }^{4}$ \\ Heriberto Rodríguez Gudiel. ${ }^{5}$
}

${ }^{1}$ Especialista en Pediatría, Jefa de Sala de Pediatría del Hospital San Felipe.

${ }^{2}$ Médico Interno de la Universidad Católica de Honduras (UNICAH). ORCID: https://orcid.org/0000-0002-8952-3320

${ }^{3}$ Subespecialista en Cirugía Pediátrica del Hospital Escuela.

${ }^{4}$ Especialista en Pediatría, pediatra de guardia del Hospital Escuela.

${ }^{5}$ Especialista Gineco-Obstetra (FACOG): Subdirección Hospital de Especialidades San Felipe; Departamento de Ginecología y Obstetricia, Hospital Escuela; Tegucigalpa, Honduras.

RESUMEN. Antecedentes: Fetus in fetu (FIF) es una rara anomalía congénita de gemelos monocigotos asimétricos, donde el gemelo parásito se desarrolla anormalmente dentro del cuerpo del gemelo huésped. Actualmente hay menos de 200 casos reportados a nivel mundial siendo este el segundo caso en Honduras. Caso clínico: Se reporta el caso de un paciente masculino, recién nacido a término en la sala de maternidad del Hospital San Felipe, quien a su nacimiento se observó distensión abdominal, hernia umbilical, hernia inguino-escrotal izquierda y tumoración en el hipocondrio izquierdo por lo que se decide realizar una radiografía anteroposterior toracoabdominal revelado un tumor en la región izquierda del abdomen con presencia de calcificaciones. Se refiriere al Hospital Escuela Universitario donde se le efectuó un ultrasonido abdominal total con reporte de masa heterogénea de $5 \mathrm{~cm}^{3}$ de volumen con componente cálcico en su interior, que tuvo que ser correlacionada con tomografía axial computarizada con reconstrucción 3D dando como resultado masa heterogenia con huesos axiales y apendiculares en su interior compatible con FIF. Se realizó intervención quirúrgica con resección de tumoración retroperitoneal y sus anexos sin secuelas ni complicaciones por lo cual se da alta médica. Conclusión: Aunque el FIF es una enfermedad muy rara, el tratamiento de elección será la resección de la masa y su pronóstico es favorable cuando la masa se ubica en el área retroperitoneal. Se puede observar que la tomografía tridimensional es una técnica de imagen útil para la diferenciación entre un FIF y un teratoma en el diagnóstico preoperatorio.

Palabras Clave: Anomalias Congénitas, Fetus in fetu, Gemelos Siameses, Gemelos Monocigóticos.
Recibido: 08-11-2019 Aceptado para publicación 25-11-2020

Dirección para correspondencia: Dr. Carlos Fernando Rosales Ordóñez

Correo electrónico: miocarlosrosales@gmail.com

Declaración de relaciones y actividades financieras y no financieras y conflictos de interés: ninguno.

\section{INTRODUCCIÓN}

Fetus in Fetu (FIF) es una rara anomalía congénita de gemelos siameses producto de embarazo diamniótico monocigotos asimétricos, donde el gemelo parásito se desarrolla anormalmente dentro del cuerpo del gemelo huésped que evoluciona normalmente a excepción del gemelo parásito, se caracteriza por estructuras altamente complejas como esqueleto u órganos. ${ }^{1-5}$

Es una condición extremadamente rara que se estima que ocurre en 1 de cada 500000 nacimientos, se han documentado menos de 200 casos de FIF a nivel mundial con un predominio 2:1 afectando más al sexo masculino., ${ }^{2,6-8}$

Este es el segundo caso en Honduras sobre FIF siendo el primero publicado por la "Revista médica hondureña" en 1999 encabezado por el Doctor José Ranulfo Lizardo. ${ }^{9}$

\section{CASO CLÍNICO}

Recién nacido a término masculino de la tercera gesta de una mujer de 31 años, procedente de la aldea Escano de Tepale, San Ignacio, Francisco Morazán. Madre del paciente con 5 atenciones prenatales y termina el embarazo por vía cesárea a causa de sospecha de embarazo gemelar más polihidramnios. Nace el 15 de septiembre del 2018, producto único, vivo, a término, macrosómico con un peso de $4.210 \mathrm{~g}$, APGAR 8 y 9 , talla $50 \mathrm{~cm}$, perímetro cefálico $38 \mathrm{~cm}$, perímetro torácico $35 \mathrm{~cm}$ y perímetro cefálico $41 \mathrm{~cm}$. Al examen físico se observó distensión abdominal, hernia umbilical (Figura 1.A), hernia inguino-escrotal izquierda y a la palpación profunda masa retroperitoneal en cuadrante superior izquierdo de bordes lisos irregulares de $5 \times 5 \mathrm{~cm}$ por lo que se solicita una radiografía toracoabdominal anteroposterior en la cual se percibe en la región izquierda una imagen calcificada simulando un esqueleto (Figura 1.B).

Es referido con sospecha de FIF al servicio de cirugía pediátrica en el Hospital Escuela Universitario el día 16 de septiembre del 2018, se solicitó ultrasonido abdominal total, realizado el 

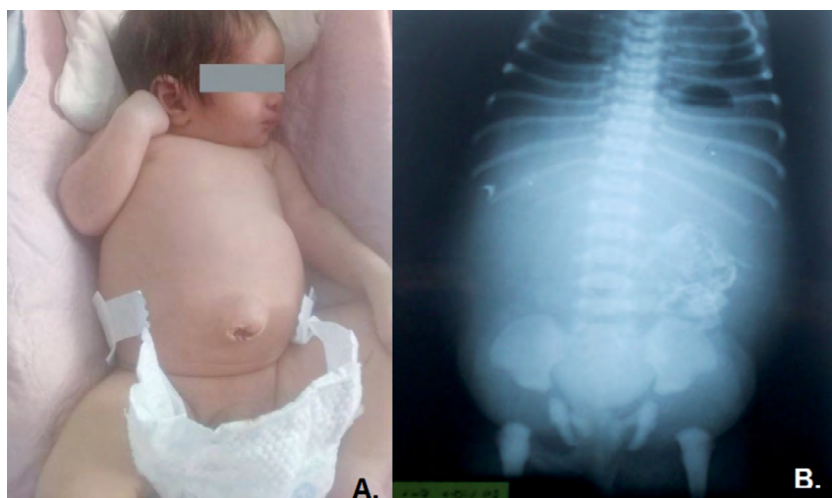

Figura 1. A. Paciente macrosómico en su segundo día de vida con hernia umbilical, abdomen distendido y perímetro abdominal aumentado en relación con el perímetro torácico y cefálico. B. Rayos x toracoabdominal en el cual se observa en la region abdominal izquierda masa radiopaca simulando esqueleto.

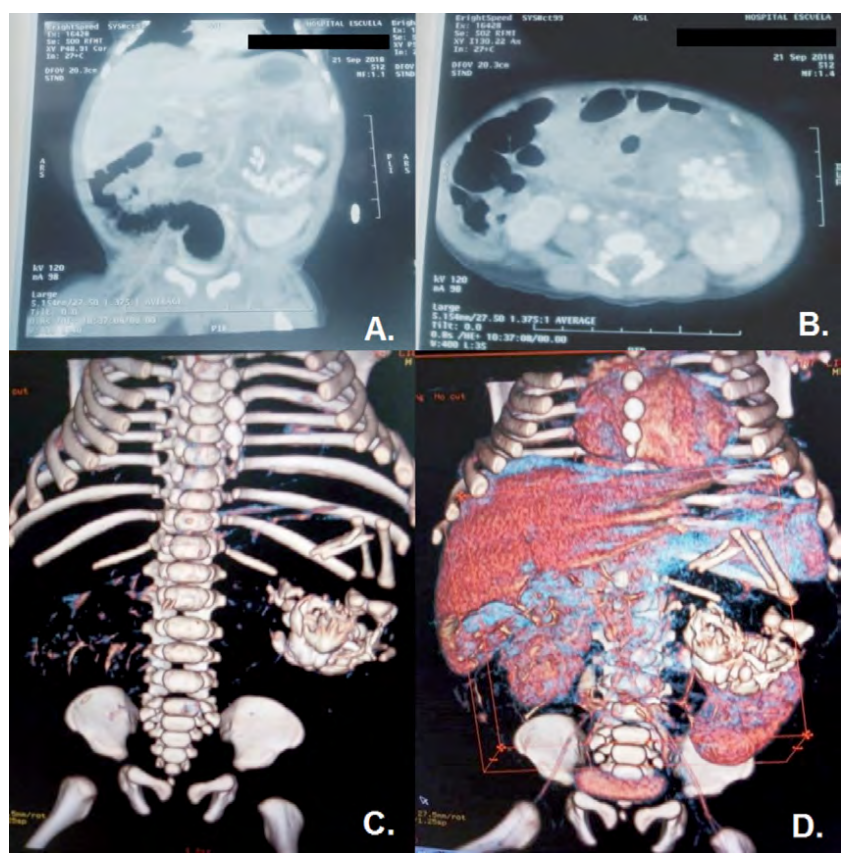

Figura 2. A. TAC abdominal de corte vertical en la cual se observa una masa hiperdensa de bordes irregulares desplazando el riñón izquierdo en sentido caudal. B. TAC abdominal de corte Horizontal a nivel de vertebral $L 4$ en la que se aprecia masa hiperdensa de alrededor de $5 \mathrm{~cm}$ de diámetro. C. TAC con reconstrucción 3D se percibe a nivel vertebral T11 a L4 masa ósea irregular en la parte izquierda del abdomen con silueta de cavidad irregular en lado derecho. D.TAC con reconstrucción 3D en la que destaca la cavidad irregular apreciando el límite superior el hígado y su límite inferior el riñón izquierdo con la masa ósea dentro de sus límites.

19 de septiembre del 2018, identificando masa heterogénea de bordes definidos con componente cálcico en su interior la cual mide $5 \mathrm{~cm}^{3}$ de volumen. Esta masa desplazó el riñón izquierdo, sin encontrar dependencia clara de estructuras adyacentes por lo que se decide correlacionar con tomografía axial computariza abdominal (TAC).

El día 21 de septiembre del 2018 se realizó TAC (Figura 2.A y 2.B) que reportó masa heterogénea de contornos bien definidos, de $5.5 \mathrm{~cm} \times 5.5 \mathrm{~cm}$ con múltiples calcificaciones en
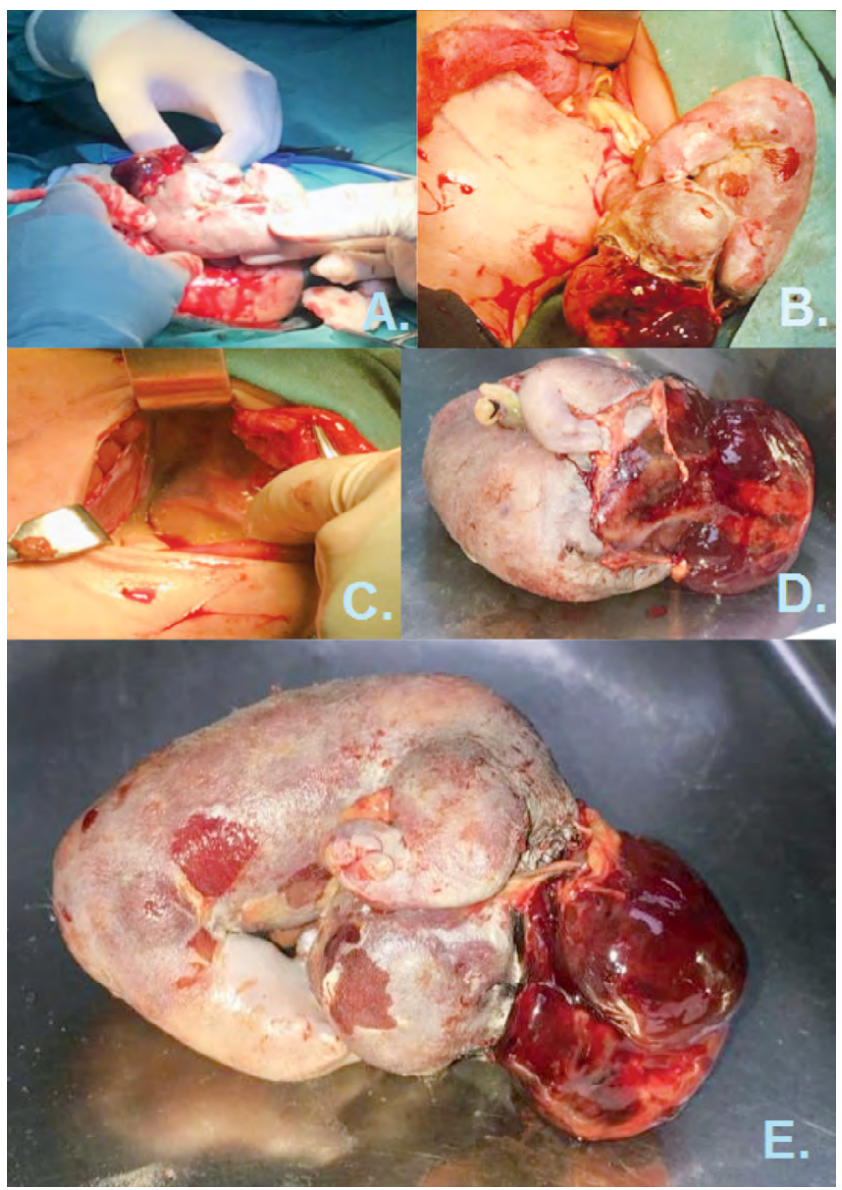

Figura 3.A. Primer abordaje de la masa fetoide de $5 \mathrm{~cm}$ de diámetro. B. Masa fetoide unido al huésped por arteria parasita que similar a un cordón umbilical también se aprecia la cara inferior de la masa aparentando una extremidad inferior con presencia digital. C. cavidad D. Cara superior de la masa fetoide en la que se identifica primera extremidad superior y agenesia cefálica. E. Cara inferior de la masa fetoide en la que se aprecia segunda extremidad superior, agenesia cefálica, lanugo y pelo.

su interior, desplazando el riñón izquierdo en sentido caudal, además con una ligera compresión de la vena cava inferior, se efectuó reconstrucción 3D del TAC (Figura 2.C) revelando huesos axiales, sin compromiso de otras estructuras adyacentes y apariencia de una silueta (Figura 2.D).

Al correlacionar las imágenes con el diagnóstico definitivo de FIF, se le practica intervención quirúrgica, el 26 de septiembre del 2018, abordándolo con una incisión paramediana en el abdomen cuadrante superior izquierdo encontrando masa de apariencia fetoide (Figura 3.A) envuelta en un saco embrionario (Figura 3.C) ubicada en la cavidad intrabdominal empujando el colon, el riñón y el uréter izquierdos, provocando las hernias umbilical e inguinal. Esta masa fetoide es irrigada por una arteria parásita que simula el cordón umbilical (Figura 3.B) anexada a la arteria mesentérica superior del huesped, la apariencia de la masa aparenta ser conformada por una sola extremidad inferior (Figura 3.B), dos extremidades superiores, (Figuras 3.D y 3.E) y una agenesia cefálica (Figuras 3.B, 3.D, 3.E). La operación se consideró satisfactoria, sin complicaciones. Se envia la masa a patología lo cual reportó 3 capas germinales ectodermo, 
mesodermo y endodermo. El huésped es dado de alta el 6 octubre de 2018 vivo y sano.

\section{DISCUSIÓN}

El primer caso documentado de FIF fue realizado por Johann Friedrich Meckel "el Joven" en su artículo "A Fetus Found In The Abdomen Of A Boy" publicado en 1808 en su libro "Teratologia". 5,6,10 Meckel "el Joven" fue un anatomista alemán nacido en Halle, trabajó como profesor de anatomía, patología y zoología en la Universidad de Halle, Alemania.

Aunque Meckel describió el primer caso de FIF no hizo una definición para esta enfermedad, siendo Rupert Allan Willis el primero en proponer una definición para esta enfermedad. Willis era un australiano patólogo que tuvo un gran impacto en el estudio de tumores. Willis definió lo que es FIF en su artículo "The structure of teratomata" difundido en 1948 como como una masa que contiene un eje vertebral y, a menudo, otros órganos y / 0 extremidades. ${ }^{4,11-14}$

Luego Rowena Spencer quien fue una médica estadounidense especializada en cirugía pediátrica, propuso en el año 2001 los criterios Spencer (Cuadro 1) en el artículo "Parasitic conjoined twins: External, internal (fetuses in fetu and teratomas), and detached (acardiacs)". 4,13,15,16

Se desconoce los orígenes y desarrollos de esta enfermedad, pero se han propuesto varias teorías. La teoría más aceptada es la "teoría del gemelo monocigótico", en la que el FIF es un gemelo diamniótico, monocoriónico y monocigótico que queda envuelto por el gemelo huésped durante el desarrollo. Otro mecanismo planteado implica la implantación de un blastocisto sobre otro blastocisto, desarrollándose posteriormente como un cuerpo de inclusión. Kaufmann y col. proponen que

Cuadro 1. Criterios de Spencer

Definición: Un feto en fetu es una masa fetiforme ubicada dentro de un feto básicamente normal, ya sea inmediatamente adyacente a uno de los sitios de unión de los gemelos unidos o asociada con el tubo neural o el sistema gastrointestinal o un derivado con una o más de las siguientes características:

(a) Encerrado dentro de un saco distinto.

(b) Cubierto parcial o completamente por piel normal.

(c) Con partes anatómicas claramente reconocibles.

(d) Unido al autositio por un pedículo que contiene unos vasos sanguíneos relativamente grandes. Se limita a una tasa de crecimiento similar a la del autositio en el que se encuentra.

Spencer R. Parasitic conjoined twins: External, internal (fetuses in fetu and teratomas), and detached (acardiacs). este proceso ocurre como resultado del síndrome de transfusión de gemelo-gemelo, con la desaparición del gemelo no dominante dando como resultado el envolvimiento del gemelo fallecido durante la tercera semana de desarrollo. Cualquiera que sea el desarrollo, es evidente que la muerte fetal y la detención del crecimiento se producen durante su desarrollo, otra característica más que lo distingue del potencial de crecimiento continuo de los teratomas maduros. ${ }^{17}$

Casi el $80 \%$ de FIF se ubica en el abdomen en el área retroperitoneal. ${ }^{7,18-23}$ Otras localizaciones menos frecuentes reportados han sido escroto, cráneo, riñones, glándulas suprarrenales, mediastino y ganglios linfáticos. Los síntomas pueden ser distensión, dificultad para alimentarse, vómitos, ictericia, retención urinaria aunque esto depende del tamaño de la masa y la ubicación. ${ }^{24}$

En la mayoría de lo casos FIF es una enfermedad benigna, el tratamiento de elección para esta enfermedad es escisión de la masa. El diagnóstico preoperatorio de FIF puede lograrse con hallazgos radiológicos siendo los rayos x útil para encontrar la presencia de esqueleto. La tomografía tridimensional como se observa en este caso sería la mejor técnica para establecer el diagnóstico ya que se puede observar de manera clara las estructuras óseas y su formación de columna axial un punto clave para su diferenciación con su principal diagnostico diferencial que es el teratoma..$^{20,21,25,26}$

\section{CONCLUSIÓN}

Aunque el FIF es una enfermedad muy rara, el tratamiento de elección será la resección de la masa y su pronóstico es favorables cuando la masa se ubica en el área retroperitoneal. Se puede observar que la tomografía tridimensional es una técnica de imagen útil para la diferenciación entre un FIF y un teratoma en el diagnóstico preoperatorio.

\section{CONTRIBUCIÓN}

GHVO, CFRO, EAT, CAGM, HRG contribuyeron substancialmente a la concepción o el diseño del estudio; a la adquisición, análisis o interpretación de los datos para el estudio; Haber redactado el trabajo o haber realizado la revisión crítica de su contenido intelectual importante; Aprobación final de la versión a ser publicada; y estar de acuerdo en ser considerado responsable de todos los aspectos del trabajo, asegurando que las preguntas relacionadas a la exactitud o integridad de cualquier parte del trabajo sean adecuadamente investigadas y resueltas. 


\section{REFERENCIAS}

1. Sitharama SA, Jindal B, Vuriti MK, Naredi BK, Krishnamurthy S, Subramania DB. Fetus in fetu: Case report and brief review of literature on embryologic origin, clinical presentation, imaging and differential diagnosis. Polish J Radiol. 2017;82:46-9.

2. Abdulraheem NT, Nasir AA, Abdur-Rahman LO, Akanbi OR, Olanrewaju MO, Alada MA, et al. Oral fetus-in-fetu: A case report. J Pediatr Surg Case Reports. 2015;3:171-3.

3. Goette M, Seitz G, Vahdad R, Obermayr F, Gummel K, Mahnken A, et al. Prenatal diagnosis of fetus in fetu pregnancy. $Z$ Geburtshilfe Neonatol. 2018;222(3):123-7.

4. Traisrisilp K, Srisupundit K, Suwansirikul S, Norasetthada T, Kosarat S, Tongsong T. Intracranial fetus-in-fetu with numerous fully developed organs. J Clin Ultrasound. 2018;46(7):487-93.

5. Narayanasamy JN, Nallusamy MA, Baharuddin ND. Fetus-in-fetu: a pediatric rarity. J Surg Case Reports. 2014;2014(2):rju001.

6. Landmann A, Calisto JL, Reyes-Múgica M, Thomas D, Malek MM. Fetusin-fetu presenting as a cryptorchid testis and abdominal mass: A report of a case and review of the literature. J Pediatr Surg Case Reports [Internet]. 2016[consultado 12 abril 2020];13:38-40.Disponible en: http://dx.doi. org/10.1016/j.epsc.2016.07.010

7. Kumar A, Paswan SS, Kumar B, Kumar P. Fetus in fetu in an adult woman. BMJ Case Rep. 2019;12(8):10-2.

8. Sewell EK, Massa-Buck B, Rubio El, Massaro AN, Badillo A, Puscasiu E, et al. Impact of prenatal diagnosis of fetus-in-fetu. J Neonatal Perinatal Med. 2017;10(3):333-8.

9. Lizardo JR, Moreno O, Figueroa C, Cardona V, Pineda A. Fetus in fetu: presentación de un caso y revisión de la literatura. Rev Méd Hondur. 1999;67(4):260-3.

10. Parquet RA. Johann Friedrich Meckel, the Younger. Acta Gastroenterol Latinoam. 2014;44(2):87.

11. Gonzalez-Crussi F. Extragonadal teratomas. 2nd ed. Washignton, D.C.: Armed Forces Institute of Pathology; 1980.

12. Kumar B, Kumar Sinha A, Kumar T, Kumar P, Sinha C, Kumar A. Is definition of "fetus in fetu" needs revision?: A case report and review of literature. J Pediatr Surg Case Reports [Internet]. 2016[consultado 23 mayo 2020];12:24-6.Disponible en: http://dx.doi.org/10.1016/j.epsc.2016.06.013

13. Padwal AD, Devi BI, Ramachandran S, Bhat DI, Shukla D, Ramu R. Occipitocervical fetus in fetu with extracalvarial extension: A case report. Pediatr Neurosurg. 2016;51(2):87-92.

14. Rupert Allan Willis. Lancet. 1980;1(8174):940.

15. Spencer R. Parasitic conjoined twins: External, internal (fetuses in fetu and teratomas), and detached (acardiacs). Clin Anat. 2001;14(6):428-44.

16. Fishkin CA. Remembering Dr. Rowena Spencer (1922-2014). J Pediatr Surg [Internet]. 2020 [consultado 23 mayo 2020];55(3):576-8. Disponible en: https://doi.org/10.1016/j.jpedsurg.2019.09.004

17. Ji $Y$, Song B, Chen S, Jiang X, Yang G, Gao X, et al. Fetus in fetu in the scrotal sac: case report and literature review. Med (Baltimore). 2015;94(32):e1322

18. Prescher LM, Butler WJ, Vachon TA, Henry MC, Latendresse T, Ignacio RC. Fetus in fetu: Review of the literature over the past 15 years. J Pediatr Surg Case Reports [Internet]. 2015[consultado 12 abril 2020];3(12):55462.Disponible en: http://dx.doi.org/10.1016/j.epsc.2015.10.006

19. Erdogdu E, Arısoy R, Kumru P, Pakay K, Demirci O, Pekin O, et al. Unusual presentation of fetus in fetu in triplet pregnancy mimicking abdominal wall defect. case Reports Perinat Med. 2015;4(2):135-6.
20. Tiwari C, Shah H, Kumbhar V, Sandlas G, Jayaswal S. Fetus in fetu: two cases and literature review. Dev period Med. 2016;20(3):174-7.

21. Aldosari SH, Alhudhaif J, Alsasi O, Farhan M, Kamel SM. Fetus in fetu with skeletal dysplasia and hyperparathyroidism. J Pediatr Surg Case Reports. 2019;44:101185

22. Ruffo G, Di Meglio L, Di Meglio L, Sica C, Resta A, Cicatiello R. Fetus-infetu: two case reports. J Matern Neonatal Med [Internet]. 2019[consultado 12 abril 2020];32(17):2812-9. Disponible en:: https://doi.org/10.1080/1476 7058.2018.1449207

23. Wang L, Long B, Zhou Q, Zeng S. Prenatal diagnosis of a "living" oropharyngeal fetus in fetu: A case report. BMC Pregnancy Childbirth. 2019;19(1):453.

24. Harigovind D, Babu SPH, Nair SV., Sangram N. Fetus in fetu .a rare developmental anomaly. Radiol Case Reports. 2019;14(3):333-6.

25. Bakr Barakat RM, Garzon S, Laganà AS, Franchi M, Ghezzi F. Fetus-infetu: a rare condition that requires common rules for its definition. Arch Gynecol Obstet [Internet]. 2020[consultado 12 abril 2020];302(6):1541-3. Epub 2019. Disponible en: https://doi.org/10.1007/s00404-019-05211-y

26. Ruiz Catena MJ, Ruiz Orpez A, Unda Freire A. Fetus in fetu versus teratoma fetiforme. An Pediatr. 2016;85(1):53-4.

ABSTRACT. Background: Fetus in fetu (FIF) is a rare congenital anomaly of asymmetric monozygotic twins, where the parasitic twin develops abnormally within the host twin's body. Currently there are less than 200 cases reported worldwide, this being the second case in Honduras. Clinical case: The case of a male patient, full-term newborn in the maternity ward of the Hospital "San Felipe", who was observed at birth, abdominal distension, umbilical hernia, left inguino-scrotal hernia and a tumor in the hypochondrium are reported. left, so it was decided to perform a thoracoabdominal anteroposterior radiography revealed a tumor in the left region of the abdomen with the presence of calcifications. She refers to the University School Hospital where a total abdominal ultrasound was performed with a report of a heterogeneous mass of $5 \mathrm{~cm} 3$ in volume with a calcium component inside, which had to be correlated with computerized axial tomography with $3 \mathrm{D}$ reconstruction, resulting in a heterogeneous mass with bones. axial and appendicular inside compatible with FIF. Surgical intervention was performed with resection of the retroperitoneal tumor and its annexes without sequelae or complications, for which the doctor was discharged. Conclusion: Although FIF is a very rare disease, the treatment of choice will be resection of the mass and its prognosis is favorable when the mass is located in the retroperitoneal area. It can be observed that three-dimensional tomography is a useful imaging technique for the differentiation between a FIF and a teratoma in the preoperative diagnosis.

Keywords: Conjoined Twins, Congenital Abnormalities, Fetus in fetu, Monozygotic Twins,. 